\title{
Multi Criteria Optimization Algorithm for Economic Dispatch Complications for Sustainable Interconnected Power System
}

\author{
Faheemullah Shaikh \\ Mehran University Centre \\ for \\ Energy \& Development, \\ Mehran University of \\ Engineering \& \\ Technology, Jamshoro, \\ Sindh, Pakistan.
}

\author{
Pervez Hameed \\ Shaikh \\ Mehran University Centre \\ for \\ Energy \& Development, \\ Mehran University of \\ Engineering \& \\ Technology, Jamshoro, \\ Sindh, Pakistan.
}

\author{
Mushtaq Mirani \\ Mehran University Centre \\ for \\ Energy \& Development, \\ Mehran University of \\ Engineering \& \\ Technology, Jamshoro, \\ Sindh, Pakistan.
}

\author{
Mohammad Aslam \\ Uqaili \\ Mehran University Centre \\ for \\ Energy \& Development, \\ Mehran University of \\ Engineering \& \\ Technology, Jamshoro, \\ Sindh, Pakistan.
}

\begin{abstract}
Power system engineers are facing difficulty in economic operation of the large interconnected power system. Economic dispatch of large interconnected power system is complex, constrained and nonlinear problem which require rigorous and complex calculations before taking any decision apropos to power dispatch. Evolutionary algorithms have been nowadays frequently used for optimum performance of nonlinear and complex systems. This paper proposed to develop an evolutionary algorithm which considers all constraints of power dispatch for economic operation of the power system.
\end{abstract}

\section{General Terms}

Economic Dispatch Problems, Computational Methods, Evolutionary Algorithms et. al.

\section{Keywords}

Power System, Economic Dispatch, Optimization, Evolutionary Algorithm

\section{INTRODUCTION}

Energy is indispensable contribution to the sustainable socioeconomic and industrial development of any country. Thus, electricity is most flexible form of energy, we use today.

Sustainable interconnected power system is a pre-requisite for transmitting power efficiently and optimally to the load centers. Escalating prices, depletion of fossil fuels and increasing environmental concerns associated with thermal power generation systems have stressed the need to use the energy resources at its optimum level [1]. On the other hand the electricity cannot be stored on large scale, therefore whatever electricity is to be generated must equate the power demand and network losses for economic operation of the power system.

Power system operation, planning and its control is complex by nature and engineers are facing challenging tasks of successful planning and economical operation of interconnected complex power system. Power system is interconnected with number of generating stations running in parallel and feeding the high voltage network and supplies electricity to the consumers. Operating such an automated complex power system is extremely multifaceted task. The Complications which the power system engineers are facing in planning and economical operation of power system are economic dispatch, environmental impacts, reliability, security, contingency planning like load shedding and generation reallocation, load flow and line losses in power system $[2,3]$.

Fig.1: Economic Dispatch Complications for Interconnected Power System

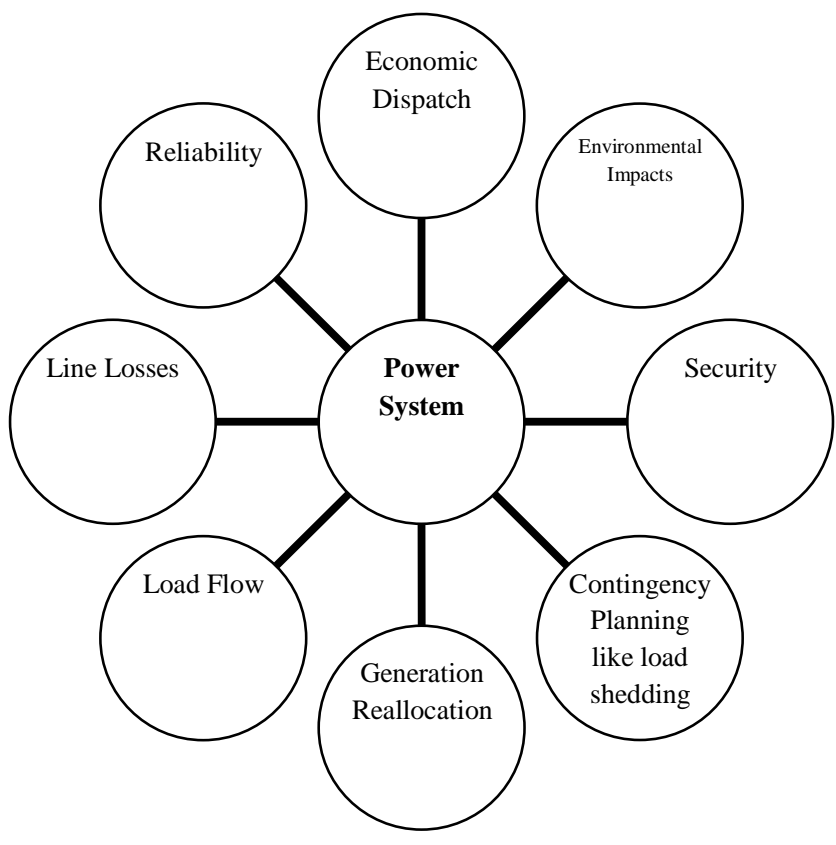

\section{POWER SYSTEM CONTROL}

Power system is combination of generation, transmission and distribution facilities. Power system is connected with multiple power generation sources including thermal, hydel and renewable energy resources. Power system control is complex task and has to make the decision based on availability of generating facilities and load demand from the different consumers. Following are the three stages of control in power system. 
Fig.2: Power System Control Stages

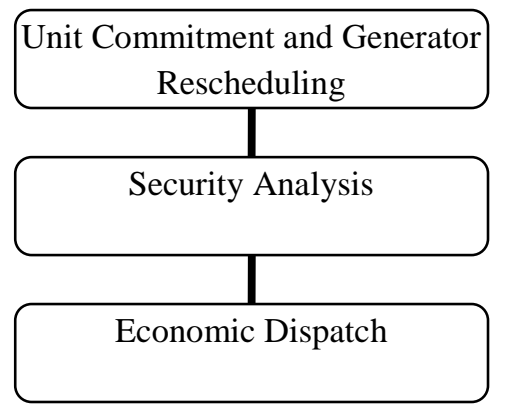

\subsection{Unit Commitment/ Generator Scheduling}

Timely ordering of the generation units, to on and off them, so as to match the load and allow the safety margins.

\subsection{Security Analysis}

Assessment of the system response to a set of contingencies and provides set of constraints that should not be violated if the system remains in secure state.

\subsection{Economic Dispatch}

Economic dispatch is short term determination of the optimal operation of generating facilities to produce energy at lowest cost to reliably serve the consumers, considering generation and transmission system operational limits. Economic dispatch calculations are carried either offline or online and degree of human intervention or supervision varies from utility to utility. In other words minute to minute loading of the generating plants, in a way to minimize the cost of generation, considering security constraints and system constraints [3].

\section{TECHNICAL ECONOMIC DISPATCH IMPEDIMENTS}

The Objective of power system is to deliver the secure and reliable electricity at least cost, least environmental pollution and least power losses. However curtailing with total fuel cost, operational effort, line losses and emissions while maintaining the reliability and security of interconnected power system are conflicting in nature. Hence economic dispatch problem is highly constrained and multi criteria optimization problem.

The Load on the power system is always changing form minute to minute, hour to hour and day to day. On the other hand equality (network) constraints and inequality (transformer on load tap changing and nodal voltages) constraints directly influence the economic operation of the interconnected power system. The constraints which directly influence the economic operation of power system are power generation, system voltage, running reserve capacity at peak loads, transformer tap settings, transmission line, sudden change in weather, fuel, acceptable low frequency, and reliability and security. Under such constrained scenario it becomes very difficult for the operator sitting in control center to dispatch the load on power system economically. Therefore it needs to adopt a multi criteria optimization approach which considers all constraints and help to economically operate the interconnected power system [1,3, 4, and 5].

\section{OPTIMIZATION ECONOMIC ALGORITHMS
BARRIER OF DISPATCH

Evolutionary algorithms are computational models, inspired by the nature and are the powerful stochastic search techniques. Evolutionary algorithms are nowadays frequently used in research fields for undertaking complex decisions under constrained scenario to reach optimum level of the performance of the system [6, 7, and 8]. Electrical power dispatch under diverse energy mix is nonlinear and complex decision making process under which it has to face lots of limitations.

[9] discussed the role of modeling and optimization in power sector for sustainable energy development scenario. $\mathrm{He}$ further identified the different aspects of comparing electricity generation and supply options such as economy, environment, health and safety, employment, energy security and social impressions. He concluded that to solve such complex constraints scenario, there needed a scientific engineering method to arrive at realistic solution. [10] designed and developed a realistic model for automatic generation control (AGC) in an interconnected power system. He developed a scheme based on genetic algorithm to control decentralized parameters like generation rate constraints, dead band and time delay imposed to the power system by governor-turbine, filters, thermodynamic process and communication channels. He come to the conclusion that neglecting above physical constraints simultaneously or in part leads to impractical and invalid results may affect the system security, reliability and integrity. [11] developed the genetic algorithm to get the biggest economic performance under constrained conditions for economical operation of the multiregional interconnected power system. He implemented his model on 14-unit system and concluded that system improved its calculation rate effectively and its economy. [12] presented the multi objective differential algorithm for nonlinear and constrained environmental, fuel cost and system loss problems. He used the Pareto optimality, which is a crowding entropy diversity measure tactic, helps to measure the crowding degree of solutions more accurately. For getting best compromise/optimize solution he employed fuzzy set theory. He implemented such optimization model on IEEE 30 and 118 bus test system and results demonstrated superior capability to solve best solution under constrained above mentioned conditions. [13] presented an idea to real time/online economical allocation of power demand at the generators. Modern generating units have cost curves that are nonlinear. Although evolutionary methods like genetic algorithm and particle swarm optimization are free from convexity assumptions but these methods converge prematurely to a local minimum solution. For tackle he introduced crazy particles to maintain momentum in search and avoid saturation. He implemented this model on test systems and results show computational efficiency, dynamic convergence, robustness and stability. [14] developed particle swarm optimization based algorithm to solve security constrained optimal active power dispatch of a localized area in large scale power system under different operating conditions. Hence minimum control variables are adjusted to operate the power system in secure and reliable state. [15] suggested different soft computing techniques like tabu search (TS), genetic algorithm (GA), hopefield neural network (HNN) and multi-layered perceptron (MLP) for solving economic dispatch problems. At the end applied these techniques to 0 n 06 and 20 generating machines and 
suggested some points to improve the performance of the soft computing technique. [16] identified that economic dispatch problem is nonlinear and complex problem, to overcome this problem, he suggested homotopy method and demonstrated that only one local minimum exists in the augmented cost function, so that the local minimum was the optimal solution of the economic power dispatch problem. [17] used decision tree (DT) by adding fuzzy logic (FL) to the unit limits and load (FLDT). A 10 unit Chilean test system is used to validate and highlight the performance of this proposition. [18] proposed key idea of combine usage of genetic algorithm and extreme learning machine technique to solve the economic dispatch problems. Modified technique helped to locate the local minima faster and therefore it would ensure faster convergence to provide more economical dispatch plans.

\section{DISCUSSION/ JUSTIFICATION}

Power economic dispatch of large interconnected power system is nonlinear and complex decision making process. Optimum performance of power system under constrained scenario has become a challenging task for power system engineers. Therefore it is required to work on optimized solution which is computational efficient, robust, stable and helps power system engineers to achieve best performance of power system under constrained scenario.

Evolutionary algorithms like genetic algorithm, particle swarm optimization, particle swarm optimization with crazy particles and fuzzy algorithms have been used by different researchers but neither of them has considered all constraints like economic dispatch, environmental impacts, reliability, security, contingency planning like load shedding and generation reallocation, load flow and line losses in power system. This paper focuses for the development of an evolutionary based algorithm while considering the constraints like simplicity, time convergent, efficient, least cost algorithm and ultimately effective which can address the cost of power generation, environmental impacts, reliability and security, contingency planning like load shedding and generation reallocation, load flow and line losses of the gridconnected power system.

To develop such an algorithm and making an allowance for multi criteria optimization to solve economic dispatch complications for sustainable grid-connected power system. It is therefore proposed to consider the all supply options like thermal, hydel and renewable power generation sources (especially solar PVs and Wind) connected with large network. Thus, enables the economic and optimal operation of the composite power system. In that case such algorithm needs to be designed simple, efficient, effective, time convergent, reliable, secure and stable. Such attributes would help power system engineer to make the optimal decision regarding economic dispatch under constrained scenario.

\section{CONCLUSION}

The expected output of this discussion paper is to solve the multi criteria based economic dispatch problems. This discussion paper concludes that the evolutionary algorithms can help very effectively in solving the multi criteria optimization problems associated with the economic dispatch of power system. The developed algorithm would consider all the constraints associated with economic dispatch and should be computationally efficient and robust, which would help to reduce the per unit cost of electricity generated, improve the reliability and security of power system network, reduce the environmental degradation, reduce the technical losses, enhance the power system capacity and improve the power quality of supply. Thus power system network would be able to be operated, controlled and planned efficiently. Another advantage would be for especially those countries which are suffering from power crisis like Pakistan where supply demand gap is very high. In that scenario to better manage the supply demand gap, power system engineers would have chance to manage the load shedding effectively.

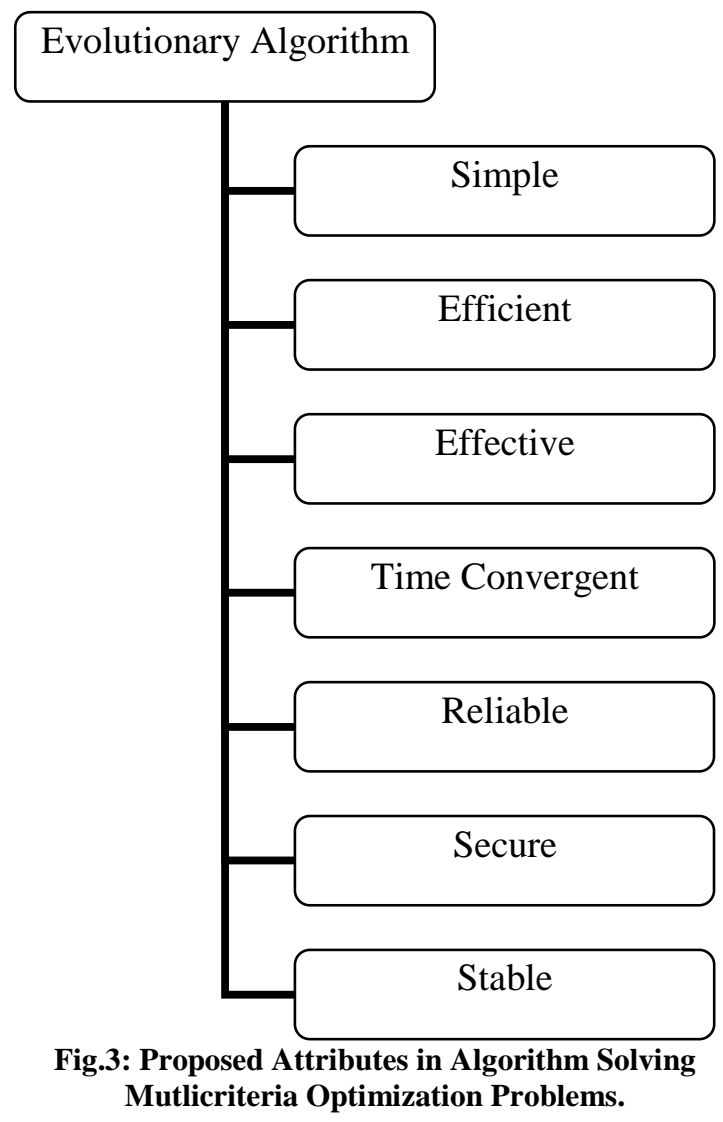

\section{REFERENCES}

[1] Harijan, Khanji; Uqaili, Mohammad A.; Memon, Mujeebuddin; Mirza, Umar "Forecasting the diffusion of wind power in Pakistan”, Journal of Energy, 2011.

[2] F. Rivas-Davalos et al, "Multiobjective Optimization Challenges in Power System: The Next Step Forward", Proceeding CERMA '07 Proceedings of the Electronics, Robotics and Automotive Mechanics Conference Pages 681-686, IEEE Computer Society Washington, DC, USA, 2007

[3] Munawar Ali Bhund, "Economic Dispatch Using Genetic Algorithm with Application to Thermal Power Station Jamshoro", Post Graduate Directorate, Mehran University of Engineering and Technology Jamshoro, Pakistan, 2011

[3] M. A. Abido, "Multiobjective Evolutionary Algorithms for Electric Power Dispatch Problem", IEEE transactions on evolutionary computation, vol. 10, no. 3, June 2006

[4] G. A. Bakare, U. O. Aliyu, G. K. Venayagamoorthy, Y. K. Shu'aib, "Genetic Algorithms Based Economic Dispatch with Application to Coordination of Nigerian Thermal Power Plants", IEEE, 2005. 
[5] Mimoun Younes, Mostefa Rahli, Lahouari Abdelhakem Koridak, "Economic Power Dispatch using Evolutionary Algorithm", Journal of ELECTRICAL ENGINEERING, 2005.

[6] Saranjeet, "Evolutionary algorithm assisted optimal placement of FACTS controllers in power system", Electrical and instrumentation engineering department, Thapar University, Patiala, Master of Engineering in Power Systems \& Electric Drives, July 2009

[7] S O. Orero and M.R. Irving, "A genetic algorithm modelling framework and solution technique for short term optimal hydrothermal scheduling", IEEE Transactions on Power Systems, 1998

[8] Amer Hasanovi, "A Simulation and Analysis Toolbox for Power Systems and Genetic Algorithm Based Damping Controller Design", Lane Department of Computer Science and Electrical Engineering, College of Engineering and Mineral Resources at West Virginia University, 2001

[9] Bazmi, A, A, Zahedi, G, "Sustainable energy systems: Role of optimization modeling techniques in power generation and supply-A review", Journal of Renewable and Sustainable Energy Reviews, 2011.

[10] Golpîra .H, Bevrani, H, Golpîra .H, "Application of GA optimization for automatic generation control design in an interconnected power system", Journal of Energy Conversion and Management, 2011

[11] Yan.G, Hongbin, S, "The Economic Operation of the Interconnect Electric Power System Based on the Genetic Algorithm", IEEE, 2010.
[12] Wua.L.H, Wangs. Y.N, Yuans. X.F, Zhou. S.W, "Environmental/economic power dispatch problem using multi-objective differential evolution algorithm", Journal of Electric Power Systems Research, 2010.

[13] Chaturvedi. K. T, Pandit .M, Srivastava. L, "Particle swarm optimization with crazy particles for nonconvex economic dispatch", Journal of Applied Soft Computing, 2008

[14] Abou El-Ela. A.A., Fetouh .T, Bishr .M.A., Saleh .R.A.F , "Power systems operation using particle swarm optimization technique", Journal of Electric Power Systems Research, 2008.

[15] H. Altun, T. Yalcinoz, "Implementing soft computing techniques to solve economic dispatch problem in power systems", Expert Systems with Applications, 2008.

[16] Jiann-Fuh Chen, Huang-Cheng Chen, Ching-Lien Huang, "The uniqueness of the local minimum for power economic dispatch problems" Electric Power Systems Research, 1995.

[17] C.A. Roa-Sepulveda, M. Herrera, B. Pavez-Lazo, U.G. Knight, A.H, "Coonick Economic dispatch using fuzzy decision trees ", Electric Power Systems Research, 2003.

[18] Hongming Yang, Jun Yi, Junhua Zhao, ZhaoYang Dong, "Extreme learning machine based genetic algorithm and its application in power system economic dispatch" Neurocomputing, 2012, In Press. 